\title{
THE CAPABILITIES OF ELECTRODISCHARGE MICRODRILLING OF HIGH ASPECT RATIO HOLES IN CERAMIC MATERIALS
}

\author{
Sebastian Skoczypiec, Magdalena Machno, Wojciech Bizoń \\ Cracow University of Technology, Institute of Production Engineering, Poland \\ Corresponding author: \\ Sebastian Skoczypiec \\ Cracow University of Technology \\ Institute of Production Engineering \\ Al. Jana Pawla II 37, 31-864 Kraków, Poland \\ phone: (+48) 12 374-37-43 \\ e-mail: skoczypiec@mech.pk.edu.pl
}

Received: 15 May 2015

Accepted: 15 July 2015

\begin{abstract}
In the first part of the article the review of ceramic materials drilling possibilities was presented. Among the described methods special attention is paid to electrodischarge drilling. This process have especially been predicted for machining difficult-to-cut electrically conductive materials. The second part consist of the results analysis of electrodischarge microdrilling of siliconized silicon carbide. The experiment involves the impact of current amplitude, discharge voltage and pulse time on the hole depth, side gap, linear tool wear and mean drilling speed. The results shows that electrodischarge drilling is a good alternative when machining inhomogeneous ceramic materials and gives possibility to drill high aspect ratio holes with relatively high efficiency (the drilling speed $>2 \mathrm{~mm} / \mathrm{min}$ ).
\end{abstract}

KEYWORDS

microdrilling, electrodischarge machining, ceramic materials, siliconized silicon carbide ( $\mathrm{SiSiC})$.

\section{Introduction}

Nowadays industry is focused on the production of smaller products with much better properties and made of new materials such as advanced ceramic and composite materials. Due to high physical properties (such as hardness, compressive strength and chemical, abrasive, corrosion, creep and temperature resistance) such advanced engineering materials are more and more applied in modern industry. The demand for macro- and micro- products and parts made of difficult-to-machine materials such as tool steel, carbides, super alloys and titanium alloys has been rapidly increasing in automotive, aerospace, electronics, optics, medical devices and communication industries. However, these materials pose many challenges to conventional machining processes (such as cutting). For example titanium alloys are susceptible to work hardening and its low thermal conductivity and higher chemical reactivity result in high cutting temperature and strong adhesion between the tool and work material leading to tool wear. Machining of such material is difficult to carry and much more expensive and in spite of their exceptional properties many of these difficult-to-machine materials seem to have limited applications. Therefore, to improve machinability and reduce costs of machining of complex shapes nontraditional machining plays an important role in nowadays manufacturing industry.

One of the most important trends in manufacturing technology deals also with the miniaturization of products and components. Micromachining is a downscaled version of existing macro level machining and is defined as shaping parts with characteristic dimensions from 1 to $1000 \mu \mathrm{m}$. Dynamic development of this machining type result from the global trend of miniaturization and demand of the market for eversmaller parts and products at better price, higher performance and with reduced power consumption. In recent years, also the need for products containing micro-holes (diameter less than $1000 \mu \mathrm{m}$ ) significantly increases. Micro-holes are used for several purpos- 
es in a lot of applications, such as inkjet printer nozzles, spinner holes, turbine blades cooling channels, diesel fuel injection nozzles, drug delivery orifices, surgical implants, optics and microelectronic components $[1,2]$. Machining of micro-holes in very hard and brittle materials by using traditional machining methods is difficult and in some cases even impossible. Therefore, the development of several drilling techniques, which have become able to fulfill the market demand in terms of miniaturization and quality take place. The selection of the proper microdrilling method depends on several aspects like the properties of workpiece material, the type, size and shape of the feature to be drilled and the target aspect ratio (the ratio of the diameter to its height). Taking into account these specification, the appropriate to specific application technology should be selected.

The purpose of this article is to describe possibilities and limitation of ceramic materials microdrilling and to present results of research on electrodischarge microdrilling process. In the first part of the article the comparison of technological features of mechanical, laser, ultrasonic, water jet, abrasive water jet, electrochemical and electrodischarge drilling was presented. The special attention was paid to use this methods to drill holes in ceramic materials. The second part of the paper consist of the results of siliconized silicon carbide ( $\mathrm{SiSiC}$ ) electrodischarge microdrilling. The experiment involves the impact of such machining parameters as current amplitude, discharge voltage and pulse time on the following technological parameters: hole depth and side gap, linear tool wear and mean drilling speed.

\section{The capabilities of ceramic material microdrilling}

Machining of ceramic and composite materials is difficult to carry out due to the nonhomogenous structure of ceramic and high abrasiveness of their reinforcing constituents. This properties usually results in damage of the workpiece and very rapid wear of the cutting tool. Therefore conventional machining processes such as mechanical turning, milling or drilling have limited application in shaping.

Drilling is a machining process used to perform holes or change dimensions and shape of holes in solid materials while the microdrilling is the drilling process adapted to machine holes with diameter less than $1 \mathrm{~mm}$. The most popular method of microdrilling is mechanical one. In this case the material is removed by applying mechanical forces with a geometrically determined cutting edge of rotating tool (drill). The drill material has to be harder than the workpiece and the cutting energy is mostly used to overcome the cohesion forces of the machined material. During machining easy to machine materials such as plastics, brass or aluminum the microdrilling is precise and repeatable process which gives possibility to obtain holes with smooth and clean walls and sharp, well defined edges. However, during drilling of high aspect ratio holes in difficult-to-machine materials the drill tip is subjected to high lateral force and the high torsional moment acts on the tool which cause bending and buckling of the drill. Therefore untimely drill wear and breakage occurs. It is worth also to underline that costs of microdrillis relatively high and geometrical limits of its miniaturization occurs (for tool diameter $100 \mu \mathrm{m}$ aspect ratios are up to ten are available).

One can state, that traditional methods of drilling are unsuitable to machine ceramic materials economically and their further development in this area is limited (the difficulty of drilling results from high toughness, additionally most of ceramic materials are harder that the materials available for use as cutting tools and therefore are unmachinable using traditional methods). The solution of these is to use nontraditional(unconventional) machining processes, which are preferred for drilling high aspect ratio holes in difficult to machine materials. In this machining methods instead traditional tool the direct form of energy (i.e. thermoelectric, electrochemical or mechanical) is utilized to remove material. In comparison to traditional processes the nontraditional machining methods can be characterized by [3]:

- capability of machining a wide spectrum of metallic and nonmetallic materials and regardless their hardness or strength,

- the mechanical properties of tool material is of no relevance (in the majority of nontradinonal machining methods there is no physical contact between the work and the tool).

These two features cause, that unconventional methods are capable to machine complex and intricate shapes in hard materials with high accuracy, good surface quality and without burrs and relatively easy miniature and micro holes and cavities can be readily produced $[2,4-8]$. In the Table 1 the characteristic of the most appropriate methods for ceramic materials drilling was presented. Among these methods the electrodischarge machining (EDM) looks like the most suitable and available technology when machining short series or prototypes an can be used with relatively small investment costs in small specialized workshops. Its detailed characteristic will be presented in the following section. 
Table 1

The characteristic of selected methods of drilling $[2,4-8]$.

\begin{tabular}{|c|c|c|}
\hline Material removal mechanism & Advantages & Disadvantages \\
\hline \multicolumn{3}{|c|}{ Mechanical drilling } \\
\hline $\begin{array}{l}\text { The material is removed by apply- } \\
\text { ing mechanical forces with a geo- } \\
\text { metrically determined cutting edge } \\
\text { (machinability is defined by me- } \\
\text { chanical properties of material) }\end{array}$ & $\begin{array}{l}\text { - precise and repeatable, } \\
\text { - smooth and clean walls of the hole, } \\
\text { - sharp, well defined hole edges. }\end{array}$ & $\begin{array}{l}\text { - tool material must be harder than the } \\
\text { workpiece, } \\
\text { - forces during machining cause bending } \\
\text { and buckling of the tool, } \\
\text { - high probability of tool breakage during } \\
\text { machining high aspect ratio holes, } \\
\text { - burr formation during machining of } \\
\text { metals, } \\
\text { - high cost of drills (especially drills with } \\
\text { diameter }<100 \mu \mathrm{m}) \text {. }\end{array}$ \\
\hline
\end{tabular}

The material is heated and removed by melting, vaporization or ablation which occurs due to interaction with focused laser beam. The machinability depends mainly on work material behaviour in respect of its reflectivity and absorptivity of laser radiation.
- can be used for drilling wide variety of - heat affected zone, metallic and nonmetallic materials regardless of their mechanical properties,

- machining without mechanical contact (no workpiece deformation),

- no tool wear,

- high drilling velocity and efficiency,

- easy to automate,

- cost-effective in series production,

- easy of drilling holes with diameter $<1$ $\mathrm{mm}$
- problems with obtaining high ratio, round and cylindrical holes,

- high equipment costs and maintenance,

- unsafe operation.

\section{Ultrasonic drilling}

The materials is machined in abrasive way. The specially designed tool oscillates with amplitude of $10-50 \mu \mathrm{m}$ at frequencies of 18 $40 \mathrm{kHz}$. The abrasive slurry is continuously fed into the working gap between the tool and the stationary workpiece. Due to oscillating tool feed in workpiece direction the abrasive particles are hammered by the tool into the machined surface and consequently they abrade the material. In some application the tool can also rotate.
- possibility of machining electrically noncondutive hard (more than 40 HRC) and brittle materials such as glass or engineering ceramics (in many cases it is difficult to find an alternative method for drilling such materials),

- high dimensional accuracy and surface quality,

- no heat generation during machining (no changes in physical properties or microstructure can be expected).

- low material removal rate comparing to other methods (especially when machining electrically conductive materials),

- not capable of machining holes and with a diameter higher than 25-30 mm with a limited depth of cut,

- high power consuming process,

- the excessive frontal and side tool wear when machining conductive materials such as steels and carbides (destroys the accuracy and leads to a considerable conicity error),

- high equipment and media costs (every job needs a special tool, the abrasive slurry should be regularly changed).

Water Jet and Abrasive Water Jet drilling

The stream of water (or abrasive and water mixture) is propelled at high pressure (180-420 $\mathrm{MPa})$ through a converging nozzle (usually diameter $0.1-0.4 \mathrm{~mm}$ ) to give a coherent jet of fluid of high speed (600-1400 m/s). The kinetic energy of the jet is converted to highpressure energy which induce high stresses exceeding the strength of machined material which results in mechanical abrasion and material removal.
- no heat affected zone (the process does not generate heat),

- precise, clean and sharp cuts that are free from burrs,

- possibility of machining various soft, hard, brittle and ductile materials at very high speeds,

- applicable for laser-reflective materials such as glass, copper, and aluminum,

- no tool (nozzle) wear when machining with water jet.
- high probability of cracking when machining brittle materials,

- high production cost due to high cost of the machine tool, the need for highly qualified operators and high maintenance requirement (not adequate for mass production),

- excessive wear of machine and tooling (nozzle) when machining with abrasive water jet,

- the abrasive water jet process is not environmentally safe. 
Table 1 [continuation]

The characteristic of selected methods of drilling $[2,4-8]$.

\begin{tabular}{|c|c|c|}
\hline Material removal mechanism & Advantages & Disadvantages \\
\hline \multicolumn{3}{|c|}{ Electrochemical drilling } \\
\hline $\begin{array}{l}\text { The material is removed by an } \\
\text { anodic electrochemical dissolution } \\
\text { process. The cathode (tool) is sep- } \\
\text { arated from the anode (workpiece) } \\
\text { by a narrow gap through which an } \\
\text { electrolyte flows (weak salt or acid } \\
\text { solution). Upon passage of electric } \\
\text { current through the gap, the anode } \\
\text { material dissolves locally. A tubu- } \\
\text { lar shaped tool, preferably made of } \\
\text { brass, copper or stainless steel is } \\
\text { used. The tool is usually insulated } \\
\text { on the entire outside surface except } \\
\text { at the tip. }\end{array}$ & $\begin{array}{l}\text { - no tool wear, } \\
\text { - high material removal rate, } \\
\text { - no heat affected zone, } \\
\text { - harder than a tool materials can be ma- } \\
\text { chined, } \\
\text { - machined surface has good quality with- } \\
\text { out residual stress and burrs, } \\
\text { - possibility of simultaneous drilling of } \\
\text { large number of deep holes of high as- } \\
\text { pect ratio. }\end{array}$ & $\begin{array}{l}\text { - only electrically conductive materials } \\
\text { can be machined, } \\
\text { - not environmental friendly process, } \\
\text { - electrolyte may cause corrosion of the } \\
\text { machining equipment, } \\
\text { - frequent failure of the tool insulation, } \\
\text { - stray removal which is an reason of ma- } \\
\text { chining delocalization, hole inaccuracy } \\
\text { and pitting occurrence. }\end{array}$ \\
\hline
\end{tabular}
Electrodischarge drilling

\begin{tabular}{|c|c|c|}
\hline $\begin{array}{l}\text { It is a thermo-electric machining } \\
\text { process in which the material re- } \\
\text { moved or eroded from the work } \\
\text { piece due to the energy from a se- } \\
\text { ries of electric discharges generated } \\
\text { between the tool electrode and the } \\
\text { workpiece immersed in a dielectric } \\
\text { medium. The electric discharges or } \\
\text { sparks remove the material by melt- } \\
\text { ing and evaporation. The dielectric } \\
\text { medium between the electrode and } \\
\text { the work piece provides the optimal } \\
\text { conditions for spark generation and } \\
\text { also flushes the debris formed in the } \\
\text { spark gap. }\end{array}$ & $\begin{array}{l}\text { - accurate, burr free and high aspect ratio } \\
\text { holes with thin walls can be drilled, } \\
\text { - applicable to machine the wide range of } \\
\text { materials, irrespective of their hardness } \\
\text { and toughness, } \\
\text { - difficult-to-machine materials (harder } \\
\text { than a tool) can be machined. }\end{array}$ & $\begin{array}{l}\text { - not applicable for materials with a bad } \\
\text { electric conductivity, } \\
\text { - significant tool wear, } \\
\text { - heat affected and recast layer occurs, } \\
\text { - low material removal rate comparing to } \\
\text { other methods, } \\
\text { - poor surface integrity. }\end{array}$ \\
\hline
\end{tabular}

\section{Electro discharge microdrilling}

In electrodischarge machining (EDM) the electrical energy is changed into thermal energy during series of discrete electric discharges which occur between two electrodes. One of the electrodes is a workpiece and the second one is a tool (Fig. 1). The material eroded from the work piece due to the energy from a series of electric discharges generated between the tool electrode and the workpiece immersed in a dielectric medium. The electric discharges or sparks remove the material by melting and evaporation. The dielectric medium between the electrode and the workpiece provides the optimal conditions for spark generation and also flushes the debris formed in the gap. The tool and the workpiece are connected to the pulse generator of with the discharge voltage from up to a few hundred volts and current ranges up to tens of amperes [9-11].

The mechanism of material removal makes EDM alternative method for shaping difficult-to-machine and electric conductive materials (hardened steel, carbides, composite materials, ceramics, etc.) [8]. De- pending on the electrode tool shape and applied machining kinematics following variants of EDM are mostly used $[8,12]$ :

- wire cutting (a wire with diameter less than $0.5 \mathrm{~mm}$ performs workpiece cutting),

- die - sinking (an electrode is mirrored in the workpiece),

- milling (shape of workpiece results from a cylindrical electrode tool trajectory),

- drilling (kinematic similar to conventional drilling, the electrode tool has tubular shape).

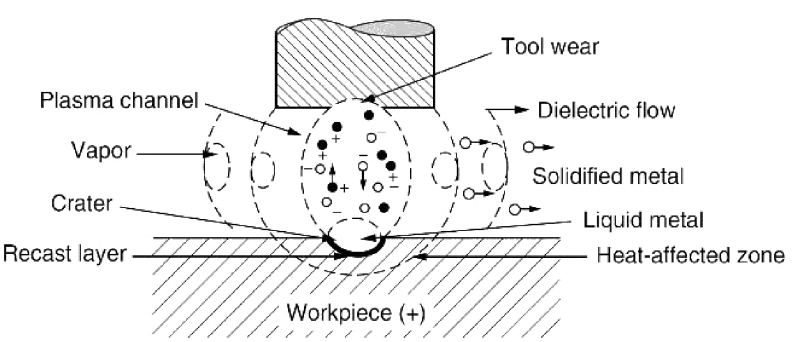

Fig. 1. Scheme of EDM material removal mechanism [3].

In EDM drilling tubular electrode tool is used and dielectric is flushed down the interior hole of the 
tube in order to remove machining debris (Fig. 2). In some cases the solid rods are used as a tool and then the dielectric is fed to the machining zone by either suction or injection through pre-drilled holes. The process gives possibility to produce regular, irregular, tapered, curved and inclined holes with typical feed rates of $0.1 \mathrm{~mm} / \mathrm{min}$ when drilling 0.1 to $0.5 \mathrm{~mm}$ diameters, leaving an overcut of 0.01 to $0.05 \mathrm{~mm}$. Typical application of EDM drilling is creating of cooling channels in turbine blades made of advanced engineering materials, fuels injectors, cutting tool coolant holes, hardened punch ejector holes, plastic molds cooling holes, wire EDM starting holes and others.

a)

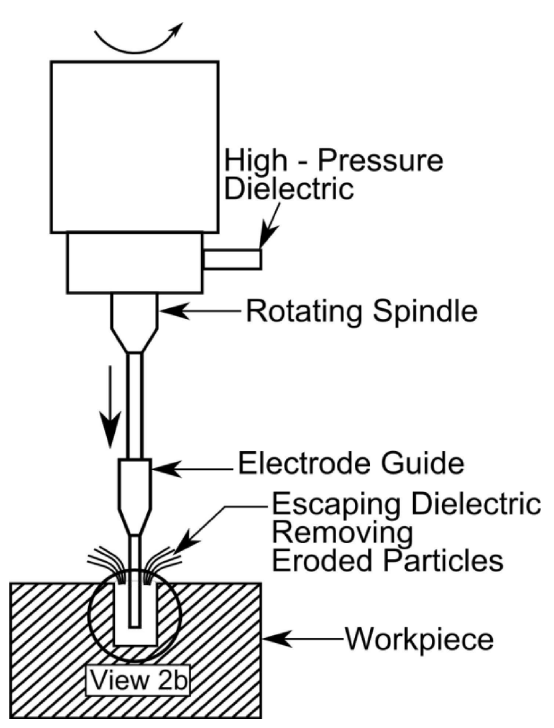

b)

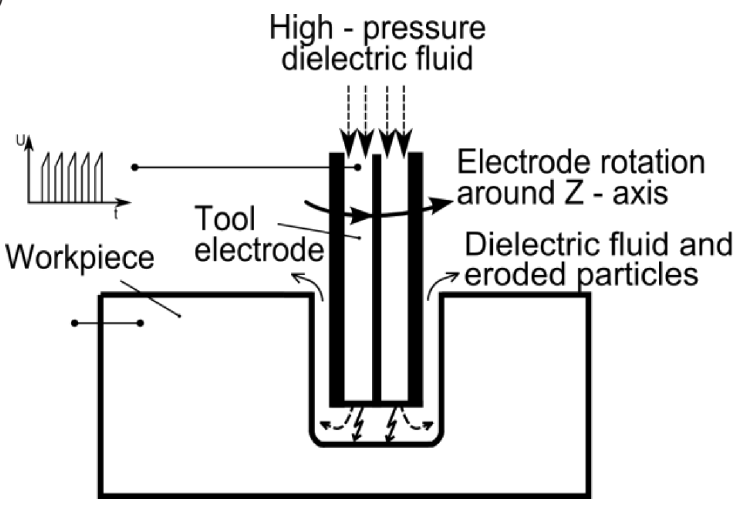

Fig. 2. Scheme of small hole EDM drilling process (a) and scheme of machining area during EDM drilling (b).

The most important issue of EDM drilling is to provide proper flushing (it is one of important variables of the process). The dielectric medium flushes the erosion products and provides an insulating medium between the electrode and workpiece (it

gives sufficient energy for discharge). In EDM drilling the deionized water is preferred as dielectric medium and in case of high aspect ratio small hole drilling high-pressure (tens of bars) flushing is used. The dielectric is pumped through the tubular, constantly rotated electrode tool, what helps in machining concentric holes (despite of tool wear) and helps in flushing process. The high pressure flushing through the centre of the electrode tends to stiffen it and dielectric being forced out of the hole produces a centric effect upon the electrode. The flushing effect and application of additional electrode guide gives possibility to penetrate much deeper than other drilling method (EDM gives possibility to obtain holes with aspect ratio up to 1/400). In many EDM drilling application of electrode with single hole problems with remove material from the center occurs (it is called carrot effect, Fig. 3a). Therefore, to overcome this effect electrodes with multiple channels were developed (Fig. 3b). Such electrode tools are usually made of graphite, brass, copper and tungsten alloys.

a)

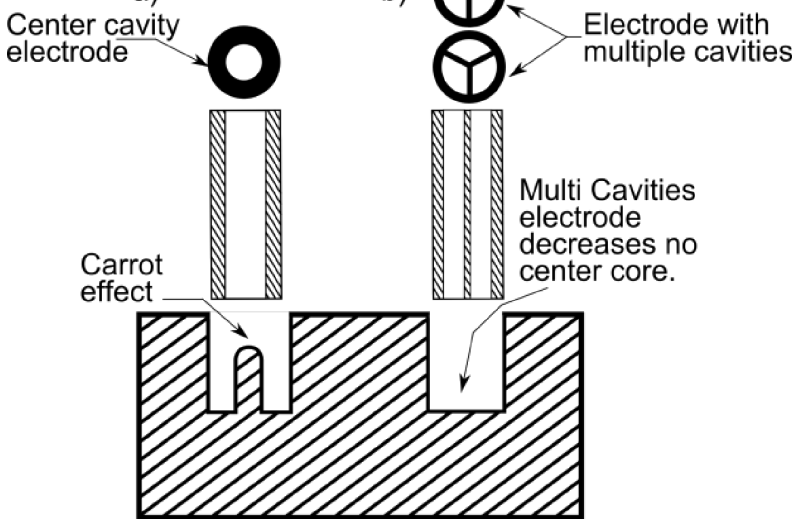

Fig. 3. Scheme of carrot effect occurring during with single channel electrode tool.

In comparison with other methods EDM drilling is characterized by such advantages as possibility to obtain accurate, burr free and high aspect ratio holes with thin walls and application to machine the wide range of materials, irrespective of their hardness and toughness (harder than a tool materials can be machined) [14-16]. However, it is worth to underline that this method has limited application for materials with a high electrical resistivity (a maximum electrical resistivity of $100 \Omega \mathrm{cm}$ ), during machining significant tool wear and low material removal rate occurs and surface layer has poor integrity and heat affected zone. 


\section{Experimental research of EDM drilling in $\mathrm{SiSiC}$ ceramic}

The research on EDM drilling was carried on at Institute of Production Engineering at Cracow University of Technology. As machined material the reaction bonded silicon-infiltrated silicon carbide ( $\mathrm{SiSiC}$ ) has been selected, which is composed of approximately 85 to $94 \% \mathrm{SiC}$ and correspondingly 15 to $6 \%$ metallic silicon $(\mathrm{Si})$. It is an important engineering ceramics because its good mechanical resistance (2000-3500 MPa to compression and $310 \mathrm{MPa}$ to tension), high hardness (HV10 $\geq 2,200 \mathrm{GPa}$ ), low density ( 3.07 to $3.15 \mathrm{~g} / \mathrm{cm}^{3}$ ), high thermal conductivity (120 to $200 \mathrm{~W} / \mathrm{mK}$ ), low coefficient of linear expansion $\left(3.6\right.$ to $4.1 \times 10^{-6} / \mathrm{K}$ at 20 to $400^{\circ} \mathrm{C}$ ) and excellent thermal shock resistance. Additionally, $\mathrm{SiSiC}$ is corrosion and wear resistant even at high temperatures. Due to its properties SiSiC ceramics can be used for extremely high mechanical and thermal loads and can be applied in applications where high temperature (up to $1350^{\circ} \mathrm{C}$ ) and wear occurs. One can state following $\mathrm{SiSiC}$ engineering application such as friction bearings and mechanical seals, for instance in pumps and drive systems, cutting tools and others applications in demanding conditions in ballistics, chemical production, energy technology, paper manufacturing and as pipe system components.

Usually the elements made of SiSiC are produced by infiltrating a formed part of silicon carbide and carbon with metallic silicon, however in some cases additional operations, such as drilling are needed. However, because of its excellent properties this material pose many challenges to conventional machining and mechanical drilling of high aspect ratio holes in such part is almost impossible, because of the extremely fast tool wear (Fig. 4) and hole surface layer properties (the drill pulls out whole grains from the workpiece and the walls are really rough).However, it is worth to note, that by infiltrating metallic silicon into the $\mathrm{SiC}$, the electrical conductivity of the matrix is largely improved, thus it can be machined by EDM [16].

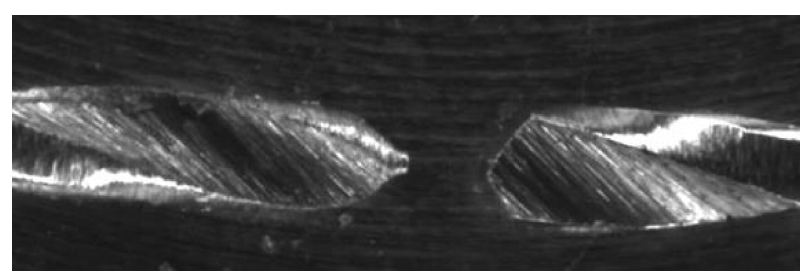

Fig. 4. Drill with the diameter of $1.2 \mathrm{~mm}$ before (on the right) and after (on the left) drilling $1 \mathrm{~mm}$ deep hole in $\mathrm{SiSiC}$ ceramics.
The EDM drilling research was carried on the machine tool designed at the Institute of Production Engineering of Cracow University of Technology (Fig. 5, detailed description in [9]). The purpose of the tests was to examine the impact of some machining parameters on hole accuracy, machining efficiency and tool wear. The research was performed according to the theory of the experiment, by three-level rotatable research plan containing following three input parameters:

- time of the pulse $\left(t_{i}: 100-500 \mu \mathrm{s}\right)$,

- current amplitude (I: $1.05-3.15 \mathrm{~A})$,

- discharge voltage (U: 20-40 V).

The output parameters was as follows:

- linear tool wear $(v)$,

- side gap $\left(S_{b}\right)$,

- drilling speed (v).

The linear tool wear $v$ was calculated, according to the following formula:

$$
v=\frac{h_{t}}{h_{h}} \cdot 100 \%,
$$

where $h_{t}$ is shortening of the electrode and $h_{h}$ is hole depth. The side gap $S_{b}$ was defined as a difference between average hole diameter $D$ (average from six measurements) and diameter of the tool $d_{t}$ divided by two:

$$
S_{b}=\frac{D-d_{t}}{2}
$$

As constant parameters shape, dimensions and material of the tool electrode (dual-channel, diameter $1 \mathrm{~mm}$, made of copper ) and dielectric fluid (deionized water) and dielectric pressure (50 bar) were assumed. In each sample with thickness $10 \mathrm{~mm}$ thorough hole was drilled, however in cases, when machining runs slowly maximal drilling time was assumed as $30 \mathrm{~min}$.

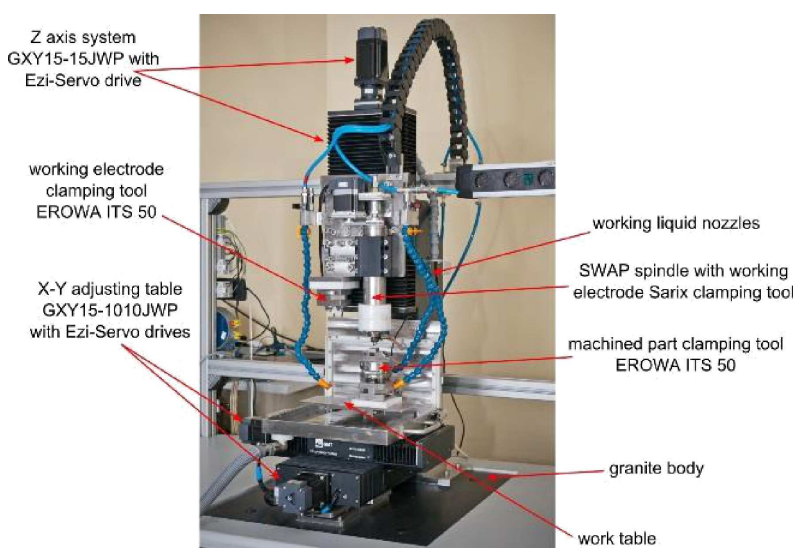

Fig. 5. EC/EDMM machine prototype designed and built at the Institute of Production Engineering of Cracow University of Technology [9]. 
As a response surface full quadratic (constant, linear, interaction, and squared terms) polynomial was selected. To calculate polynomial coefficients and perform regression analysis the Matlab software was used. From presented in Figs. 6-9 relations following conclusions can be formulated:

- the linear tool wear mainly depends on current amplitude and pulse time (wear increases with current amplitude and pulse time increase) and is in range of $1 \%$ to $5 \%$ (the maximal value of linear tool wear reach about $5 \%$ for current amplitude higher than 2.5-3 A),

- the speed of SiSiC ceramic drilling reach up to $2 \mathrm{~mm} / \mathrm{min}$ and depends mainly on discharge voltage and current amplitude (Fig. 7) and the pulse time has rather an insignificant effect on the drilling speed (Fig. 8),

- the side gap $S_{b}$, which indicates the drilling accuracy, is relatively high and is in range of 50 to $100 \mu \mathrm{m}$ and depends mainly on discharge voltage and current amplitude (Fig. 9). Such high values of $S_{b}$ are related with $\mathrm{SiC}$ grain size.

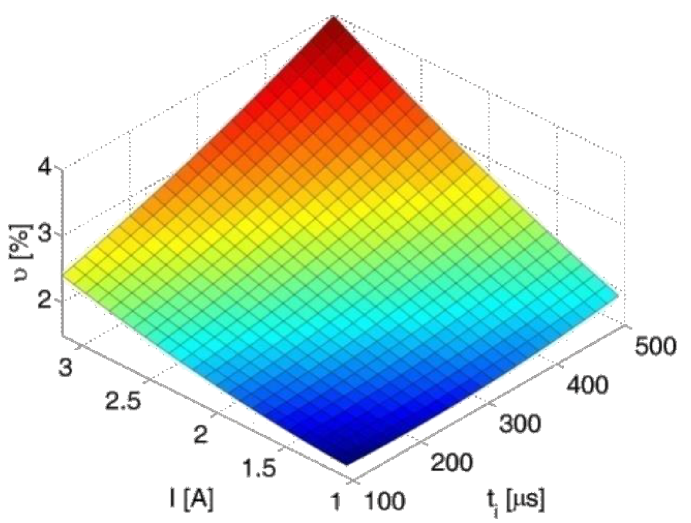

Fig. 6. Relationship between linear tool wear $v$, current amplitude $I$ and time of the impulse $t_{i}, U=30 \mathrm{~V}$.

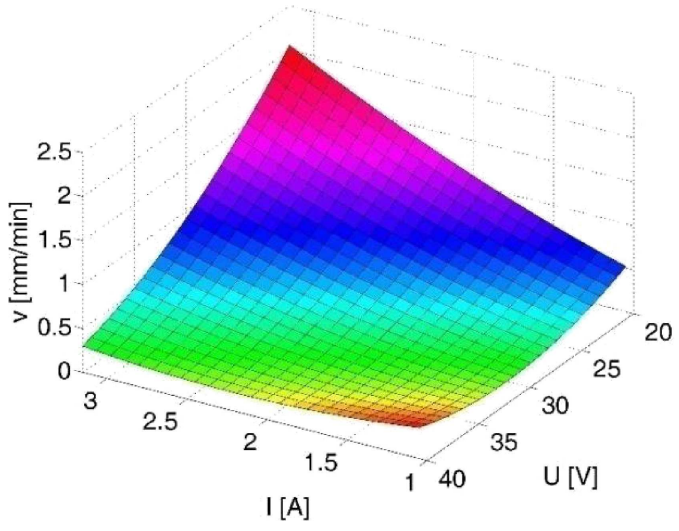

Fig. 7. Relationship between drilling speed $V$, current amplitude $I$ and discharge voltage $U, t_{i}=300 \mu \mathrm{s}$.

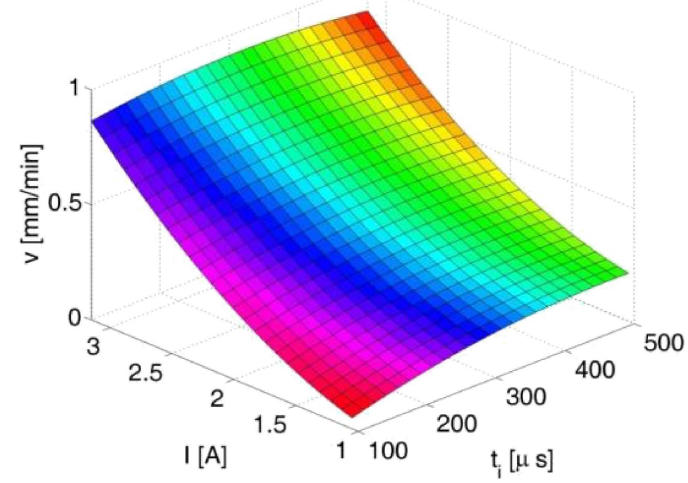

Fig. 8. Relationship between drilling speed $V$, current amplitude $I$ and time of the impulse $t_{i}, U=30 \mathrm{~V}$.

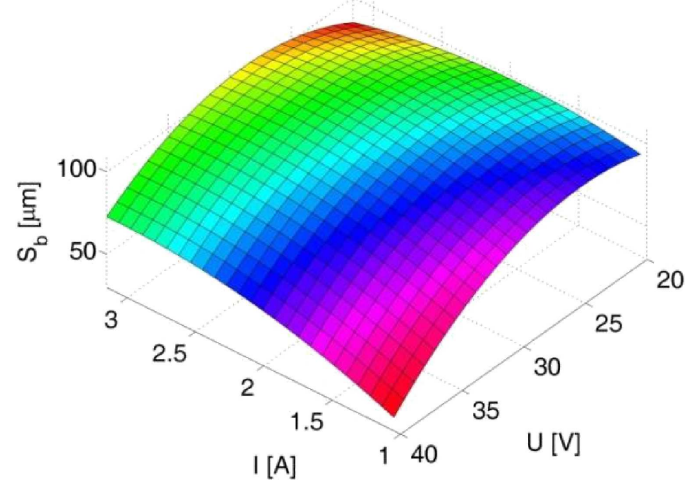

Fig. 9. Relationship between side gap Sb, current amplitude $I$ and discharge voltage $U, t_{i}=300 \mu \mathrm{s}$

It is worth to underline that in case when deionized water is applied as working fluid the electrodischarge erosion is accompanied by electrochemical dissolution. The deionized water can be treated as weak electrolyte therefore, one can state that material is removed by simultaneous interaction of electrochemical dissolution and electrical discharges on the workpiece surface. When discharge voltage is low, the electrochemical removal component takes important part in material removal, what cause that side gap is higher (Fig. 9) and SiC grains more easily flows out from the machining area. It results in more stable machining and increase drilling velocity (Fig. 7). The share of discharges in the removal process is directly connected with the intensity of the electrochemical reaction, which change of electrolyte properties (temperature and gas amount increase). When discharge voltage and current amplitude are high $(U>35 \mathrm{~V}$, $I>2 \mathrm{~A}$ ) the passing current heats the water which begins to boil what decreases its conductivity and decreases speed of the electrochemical dissolution. The result is a decrease in the gap thickness and the material is removed in a typical way for the EDM process. 
The shape of holes drilled in $\mathrm{SiSiC}$ ceramic with extreme values of current amplitude $I$ and discharge voltage $U$ were presented in Fig. 10. One can state that holes machined with $U=20 \mathrm{~V}$ have more regular shape. This results from bigger side gap for this value of $U$ which causes that dielectric flow is more uniform. On the edges of the holes machined with maximal voltage $(U=40 \mathrm{~V})$ chipping also occurs. Regardless of applied value of current amplitude the difference in the drilling speed for machining with extreme values of voltage are significant (Fig. 11). For $I=1.05 \mathrm{~A}$ speed of drilling with $U=20 \mathrm{~V}$ is almost three times higher and for $I=3.15 \mathrm{~A}$ is more than six time higher than for $U=40 \mathrm{~V}$.
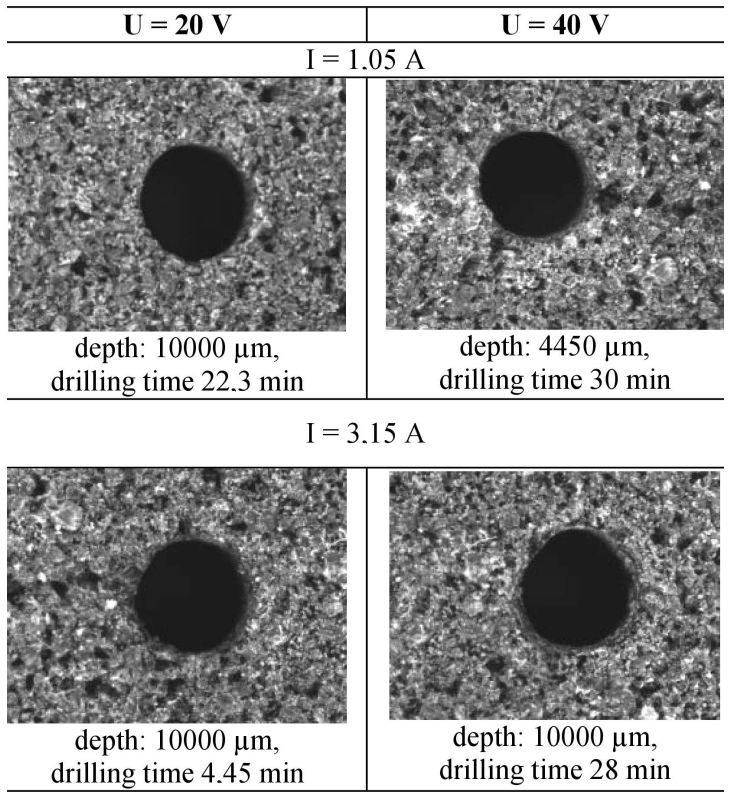

Fig. 10. Photographs of the holes drilled in $\mathrm{SiSiC}$ ceramic with extreme values of current amplitude $I$ and discharge voltage $U$ used during the tests, pulse time $t_{i}=300 \mu \mathrm{s}$, thickness of the sample: $10000 \mu \mathrm{m}$.

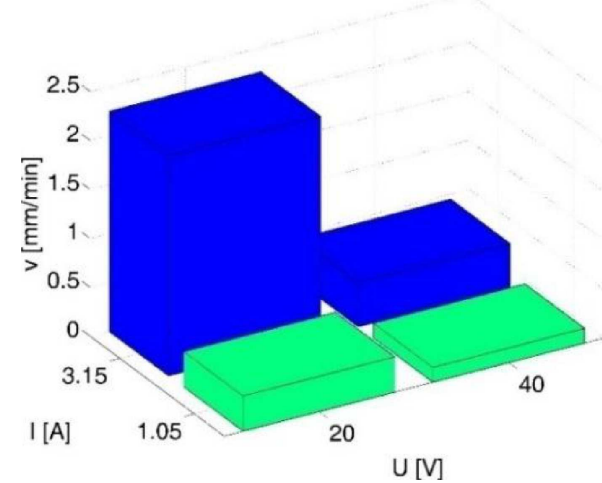

Fig. 11. Speed of drilling in $\mathrm{SiSiC}$ ceramic with extreme values of current amplitude $I$ and discharge voltage $U$ used during the tests, pulse time $t_{i}=300 \mu \mathrm{s}$.

\section{Conclusion}

In the first part of the article the review of ceramic materials microdrilling possibilities was presented. Among the described methods special attention is paid to electrodischarge drilling. These process have especially been predicted for machining high aspect ratio holes in difficult-to-cut electrically conductive materials. The second part of the paper consist of the results analysis of electrodischarge microdrilling of siliconized silicon carbide ( $\mathrm{SiSiC}$ ). The experiment involves the impact of such machining parameters as current amplitude, discharge voltage and pulse time on the following technological parameters: hole depth and side gap, linear tool wear, mean drilling speed. The analysis of relationship between input and output data, show that main input parameters are current amplitude and discharge voltage (the pulse time has only significant effect on the tool wear). Detailed discussion of results led to the conclusion that during machining electrodischarge erosion is accompanied by electrochemical dissolution and machining in carried out in hybrid way (electrochemical discharge machining).

Research carried out proved, that electrodischarge machining is a good alternative when drilling conductive inhomogeneous ceramic materials. One can state that area of application of this method is to drill high ratio through holes with relatively high efficiency (the drilling speed can reach more than $2 \mathrm{~mm} / \mathrm{min}$ ). However it is high energy consuming process, therefore the application of electrodischarge drilling should be considered only in cases when use of traditional methods is uneconomical or impossible.

\section{References}

[1] DeFu Liu, YongJun Tang, W.L. Cong, A review of mechanical drilling for composite laminates, Composite Structures, 94, 1265-1279, 2012.

[2] Sen M., Shan H.S., A review of electrochemical macro- to micro-hole drilling processes, International Journal of Machine Tools \& Manufacture 45, 137$152,2005$.

[3] El-Hofy H., Advanced Machining Process. Nontraditional and hybrid machining process, Alexandria University, Egypt, 2005.

[4] Puertas I., Luis C.J., A study on the electrical discharge machining of conductive ceramics, Journal of Materials Processing Technology, 153-154, 10331038, 2004. 
[5] Samant A.N., Dahotre N.B., Laser machining of structural ceramics - A review, Journal of the European Ceramic Society, 29, 969-993, 2009.

[6] Rupinder Singh, Khamba J.S., Ultrasonic machining of titanium and its alloys: A review, Journal of Materials Processing Technology, 173, 125-135, 2006.

[7] Alberdi A., Suárez A., Artaza T., Escobar-Palafox G. A., Ridgway K., Composite Cutting with Abrasive Water Jet, Procedia Engineering, 63, 421-429, 2013.

[8] Rajurkar K.P., Sundaram M.M., Malshe A.P., Review of Electrochemical and Electrodischarge $M a$ chining, Procedia CIRP, 6, 13-26, 2013.

[9] Skoczypiec S., Ruszaj A., A sequential electrochemical-electrodischarge process for micro part manufacturing, Precision Engeneering, 38, 680-690, 2014.

[10] Kunieda M., Lauwers B., Rajurkar K.P., Schumacher B.M., Advancing EDM through Fundamental Insight into the Process, CIRP Annals - Manufacturing Technology, 64-87, 2005.

[11] Pham D.T., Dimov S.S., Bigot S., Ivanov A., Popov K., Micro-EDM - recent developments and research issues, Journal of Materials Processing Technology, 149, 50-57, 2004.

[12] Ho K.H., Newman S.T., State of the art electrical discharge machining (EDM), International Journal of Machine Tools \& Manufacture, 43, 1287-1300, 2003.

[13] Pham D.T., Dimov S.S., Bigot S., Ivanov A., Popov K., Micro EDM drilling accuracy study, Advances in Integrated Design and Manufacturing in Mechanical Engineering, 281-294, 2005.

[14] D'Urso G., Maccarini G., Ravasio C., Process performance of micro-EDM drilling of stainless steel, The International Journal of Advanced Manufacturing Technology, 72, 9-12, 1287-1298, 2014.

[15] Jahan M.P., Rahman M., Wongb Y.S., A review on the conventional and micro-electrodischarge machining of tungsten carbide, International Journal of Machine Tools \& Manufacture, 51, 837-858, 2011.

[16] Clijsters S., Liu K., Reynaerts D., Lauwers B., EDM technology and strategy development for the manufacturing of complex parts in SiSiC, Journal of Materials Processing Technology, 210, 631-641, 2010. 